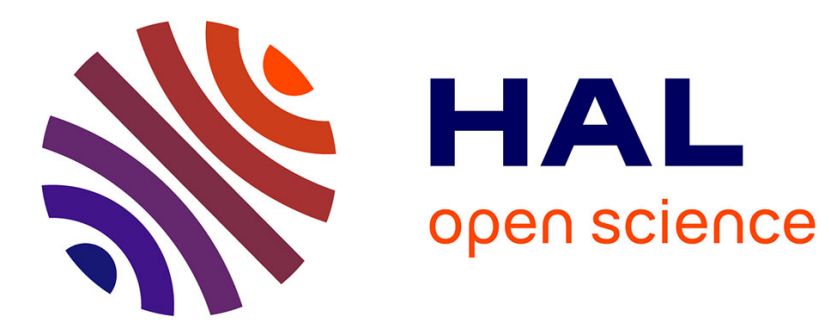

\title{
On the glassy nature of random directed polymers in two dimensions
}

\author{
Marc Mézard
}

\section{To cite this version:}

Marc Mézard. On the glassy nature of random directed polymers in two dimensions. Journal de Physique, 1990, 51 (17), pp.1831-1846. 10.1051/jphys:0199000510170183100 . jpa-00212493

\section{HAL Id: jpa-00212493 https://hal.science/jpa-00212493}

Submitted on 1 Jan 1990

HAL is a multi-disciplinary open access archive for the deposit and dissemination of scientific research documents, whether they are published or not. The documents may come from teaching and research institutions in France or abroad, or from public or private research centers.
L'archive ouverte pluridisciplinaire HAL, est destinée au dépôt et à la diffusion de documents scientifiques de niveau recherche, publiés ou non, émanant des établissements d'enseignement et de recherche français ou étrangers, des laboratoires publics ou privés. 


\title{
On the glassy nature of random directed polymers in two dimensions
}

\author{
Marc Mézard \\ Laboratoire de Physique Théorique de l'Ecole Normale Supérieure (*), 24 rue Lhomond, 75231 \\ Paris Cedex 05, France
}

(Received on April 2, 1990, accepted on May 16, 1990)

\begin{abstract}
Résumé. - Nous étudions numériquement des polymères dirigés dans un potentiel aléatoire en $1+1$ dimensions. Nous introduisons deux copies du polymère, couplées par une interaction thermodynamique locale. Nous montrons que le système est instable sous l'effet d'une répulsion arbitrairement faible entre les deux copies. Ceci suggère une similitude avec une phase verre de spin, avec plusieurs "vallées ", où les différences d'énergie libre entre vallées croissent comme $t^{\tilde{\omega}}$, où $t$ est la longueur du polymère et $\tilde{\omega}$ est probablement égal à $1 / 3$. L'effet d'un champ électrique transverse est étudié en détails et on démontre l'existence pour la susceptibilité correspondante d'importantes fluctuations d'un échantillon à l'autre. Les résultats des simulations sont comparés à des calculs analytiques utilisant la représentation de ce problème en termes de mécanique quantique et l'Ansatz de Bethe.
\end{abstract}

\begin{abstract}
We study numerically directed polymers in a random potential in $1+1$ dimensions. We introduce two copies of the polymer, coupled through a thermodynamic local interaction. We show that the system is unstable versus an arbitrary weak repulsion of the two copies. This suggests a similarity with a spin glass phase, with several " valleys", where the typical differences of the free energies of the valleys grow like $t^{\tilde{\omega}}$, where $t$ is the length of the polymer and $\tilde{\omega}$ is probably equal to $1 / 3$. The effect of a transverse electric field is studied in details showing the existence of strong fluctuations from sample to sample in the corresponding susceptibility. The results of the simulations are compared to analytic computations using the quantum mechanical formulation of the problem and the Bethe Ansatz.
\end{abstract}

\section{Introduction.}

The study of directed polymers in a random potential is an active research topic which is important in several respects [1-6]. Besides the polymers themselves it is related to interface fluctuations and pinning [2], spin glasses [3], crystal growth [4], the random stirred Burgers equation in fluid dynamics [5], and quantum mechanics in a time-dependent random potential [6]. In this paper we shall be particularly interested in its relation with spin glasses, since this system might well constitute a kind of «baby spin glass » problem. This relation with spin

${ }^{*}$ ) Unité propre du Centre National de la Recherche Scientifique, associée à l'Ecole Normale Supérieure et à l'Université de Paris Sud. 
glasses was particularly emphasized by Derrida and Spohn [3]. Solving the mean field theory of the polymer problem, i.e. the problem of polymers on the Cayley tree, they found a rather simple spin glass structure. This system has a low temperature phase with broken ergodicity and many pure states, where the overlaps between different states (the fraction of common monomer positions) vanish. This behaviour is exactly identical to the random energy model [7] which has been shown to be a kind of simplest spin glass model [8], displaying most of the properties of the Sherrington-Kirkpatrick model [9]. For finite dimensional random directed polymers in $d+1$ dimensions the situation is somewhat less clear. A phase transition has been shown to exist for $d>2$ [10] and is found numerically for $d=2$ [11] but the nature of the low temperature phase has been explored only through $1 / d$ expansions [12].

Here we want to study this problem starting from the other end, i.e. in $1+1$ dimensions. The problem is easily formulated : in a two dimensional "space time" with coordinates $(x, \tau)$ we consider all oriented walks $x(\tau), \tau \in[0, t]$ with $x(0)=0$, and $x(t)=y$. The partition function of this system at temperature $T=1 / \beta$ is :

$$
\begin{gathered}
Z(y, t)=\int_{x(0)=0, x(t)=y} \mathrm{~d}[x] \mathrm{e}^{(-\beta H[x])} \\
-\beta H[x]=-1 / 2 \int_{0}^{t}\left(\frac{\mathrm{d} x}{\mathrm{~d} \tau}\right)^{2} \mathrm{~d} \tau-\beta \int_{0}^{t} V(x(\tau), \tau) \mathrm{d} \tau
\end{gathered}
$$

where we have added to the usual Wiener measure for random walks an extra space time dependent random potential $V(x, \tau)$. We want to study the case where the potential is of the white noise type with local correlations (throughout this paper a bar denotes the average over various samples and \langle\rangle denotes the thermal averages):

$$
\left.\overline{V(x, \tau) V\left(x^{\prime}, \tau^{\prime}\right.}\right)=\delta\left(x-x^{\prime}\right) \delta\left(\tau-\tau^{\prime}\right) .
$$

(A simpler model with nonlocal correlations can be analysed in great details [13].)

The total partition function for walks originating at $(0,0)$ is :

$$
Z(t)=\int Z(y, t) \mathrm{d} y .
$$

The thermodynamics of the problem is given by the quenched free energy:

$$
F=-T \overline{\log Z(t)}
$$

and another interesting quantity is the probability density of the position of the end point :

$$
P(x, t)=\frac{Z(x, t)}{Z(t)} .
$$

This problem can be handled through numerical simulations, where transfer matrix techniques enable an exact computation of $Z(x, t)$ for systems of sizes up to several thousands time steps [14]. It can also be studied analytically through a mapping to the quantum mechanical problem of which (1) is a path integral representation and through the use of the replica method [6]. Many efforts have already been devoted to this study; it is expected that this system does not have a phase transition and is always in its low temperature phase. Furthermore the typical extension of the walk, characterized by :

$$
\overline{\left\langle x^{2}\right\rangle}=\int \bar{P}(x, t) x^{2} \mathrm{~d} x
$$


is known to scale as $\overline{\left\langle x^{2}\right\rangle} \sim t^{2 \nu}$ where several arguments, both numerical $[2,14,1]$ and analytical $[5,6,15]$, indicate that the exponent $\nu$ is equal to $2 / 3$.

Our aim is to study the nature of this phase, and especially to try to analyse if it shares some aspects of a spin glass phase. The approach will be mostly numerical. In the next section we analyse a system of two copies of the polymer coupled through a repulsive local interaction. We also study the effect of weak transverse electric field, coupled to the endpoint position of the polymer. Section 3 draws some comparison with some analytic Ansatz for $Z(x, t)$ recently proposed by Parisi [15]. Section 4 contains some speculations on the physical nature of this phase as it might be guessed from the preceeding numerical simulations.

\section{Numerical simulations.}

In the mean field theory of spin glasses, a standard method to characterize the spin glass phase is to compute the distribution of overlaps $P(q)[16,9]$. To compute $P(q)$ for one given sample we introduce two identical copies $x(\tau)$ and $y(\tau)$ of the system (with the same realization of the potential) which are uncoupled, and compute the distribution of

$$
q=\frac{1}{t} \int_{0}^{t} \delta(x(\tau)-y(\tau)) \mathrm{d} \tau
$$

Numerically it is easier to compute the first few moments of $\overline{P(q)}$; we find that $\langle q\rangle$ goes to a finite and nonzero limit $q_{0}$ (which depends on the temperature) when $t \rightarrow \infty$, but the second connected moment $\overline{\left\langle q^{2}\right\rangle-\langle q\rangle^{2}}$ seems to go to zero in the large time limit, suggesting a trivial $\overline{P(q)}$ function : $\overline{P(q)}=\delta\left(q-q_{0}\right)$.

However it is well known that a system can have several pure states and a trivial $\overline{P(q)}$ function $[17,18]$. This happens typically when the free energy differences between the states grow like $t^{\gamma}$, with $0<\gamma<1$, in the limit of large volumes. (To get a nontrivial $P(q)$ one needs $\gamma=0$.) Recently Parisi and Virasoro [18] have pointed out that the existence of several states and of replica symmetry breaking effects in spin glasses should be analysed through the reactivity to a thermodynamic coupling of several copies of the system. This kind of approach has already been used in the numerical study of three dimensional spin glasses [22]. Specifically we introduce two identical copies of the system, which are coupled through a delta function potential of strength $\varepsilon$. The Hamiltonian we consider is therefore :

$$
-\beta H_{2}[x, y, \varepsilon]=-\beta(H[x]+H[y])+\beta \varepsilon \int_{0}^{t} \delta(x(\tau)-y(\tau)) \mathrm{d} \tau
$$

(for $\varepsilon>0$ the coupling between the two copies is attractive while for $\varepsilon<0$ it is repulsive). The averaged free energy is

$$
F_{2}(\varepsilon)=-\frac{1}{\beta} \log \left(\int_{x(0)=0} \mathrm{~d}[x] \int_{y(0)=0} \mathrm{~d}[y] \mathrm{e}^{-\beta H_{2}[x, y, \varepsilon]}\right)
$$

and the overlap between the two copies is :

$$
q(\varepsilon)=-\frac{\mathrm{d} F_{2}}{\mathrm{~d} \varepsilon}
$$

We have computed numerically the average overlap $q(\varepsilon)$ for various values of $\varepsilon$ using a transfer matrix method. We use a discretized version of (1) where the points lie on a 
two dimensional grid $(x, t) \in Z \times N$ and the partition function for pairs of polymers coupled as in (8) arriving at points $x, y$ at time $t$, satisfies the recursion relation:

$$
\begin{aligned}
Z(x, y, t+1)=\mathrm{e}^{-\beta(V(x, t+1)+V(y, t+1))} \sum_{\xi= \pm 1} \sum_{\eta= \pm 1} Z(x+\xi, y+\eta, t) \times \\
\times\left[\left(1-\delta_{x, y}\right)+\mathrm{e}^{\beta \varepsilon} \delta_{x, y}\right] .
\end{aligned}
$$

In order to compute $q$ we introduce the partition function $\tilde{Z}(x, y, q, t)$ which sums over pairs of paths arriving respectively at the points $x$ and $y$, with a fixed overlap $q$. The first moment in $q$ of $\tilde{Z}, Y(x, y, t)=\sum_{q=0}^{\infty} q \tilde{Z}(x, y, q, t)$ satisfies :

$$
\begin{aligned}
Y(x, y, t+1)=\mathrm{e}^{-\beta(V(x, t+1)+V(y, t+1))} \sum_{\xi= \pm 1} \sum_{\eta= \pm 1}\left[\left(1-\delta_{x, y}\right) Y(x+\xi, y+\eta, t)+\right. \\
\left.+\delta_{x, y}(Y(x+\xi, y+\eta, t)+Z(x+\xi, y+\eta, t))\right] .
\end{aligned}
$$

Eventually one gets :

$$
q_{t}(\varepsilon)=\frac{1}{t} \frac{\sum_{x, y} Y(x, y, t)}{\sum_{x, y} Z(x, y, t)} .
$$

Equations (11), (12) have been iterated with gaussian local potential of mean 0 and variance 1. Most of the simulations were carried out at $\beta=7$, and some of them at $\beta=10$. The sizes ranged up to $t=255$. The results are as follows (see Fig. 1): for $\varepsilon>0$ there are very little finite size effects and nothing spectacular happens, in the sense that $\lim q_{t}(\varepsilon)$ is a smooth function $q(\varepsilon)$. (For $\beta=7, q(0) \sim 0.87$.) For $\varepsilon<0$ (repulsive $t \rightarrow \infty$

interaction) the overlap $q_{t}(\varepsilon)$ decreases. But there is a strong finite size effect : the decrease is much sharper for large systems. In fact the data for $q_{t}(\varepsilon)$ can be plotted with the scaling form $q_{t}(\varepsilon)=q\left(\varepsilon t^{1-\tilde{\omega}}\right)$. Figure $1 \mathrm{~b}$ shows this scaling form, with $\tilde{\omega}=1 / 3$. This value has been chosen because of some arguments which will be exposed in the last section. From the numerical data of $q_{t}(\varepsilon)$ one cannot determine $\tilde{\omega}$ with high precision, but it is found to be in the range $0.2-0.4$. There are still some finite size effects at $t=255$ which make it difficult to give a much more precise determination of $\tilde{\omega}$.

From this scaling we find that :

$$
\begin{aligned}
& \lim _{\varepsilon \rightarrow 0^{-}} \lim _{t \rightarrow \infty} \overline{q_{t}(\varepsilon)}=0 \\
& \lim _{\varepsilon \rightarrow 0^{+}} \lim _{t \rightarrow \infty} \overline{q_{t}(\varepsilon)}=\overline{q(0)} \sim 0.87 .
\end{aligned}
$$

Therefore the free energy $F_{2}(\varepsilon)$ is nonanalytic at $\varepsilon=0$. This shows that this phase has a nontrivial structure with the coexistence of several pure states. It has recently been proposed that such an effect could be present [15], this will be discussed in the next section. We shall expand on a possible interpretation of these results in the last section.

Besides the coupling between two copies, another kind of perturbation one can add to the pure system is a field conjugate to the endpoint position $x(t)$ of the polymer. This is useful in order to study the distribution $P(x, t)$ defined (5). This field $h$ adds a term $-h x(t)$ to the 


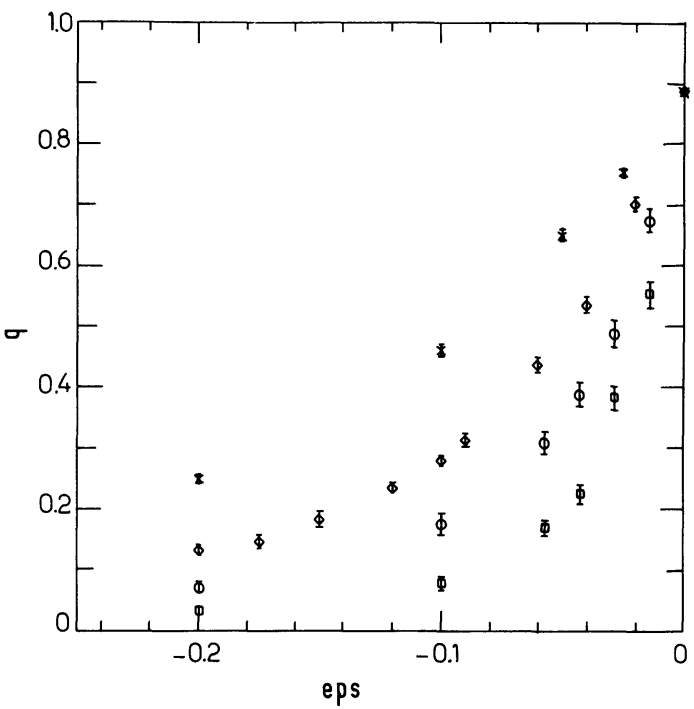

a)

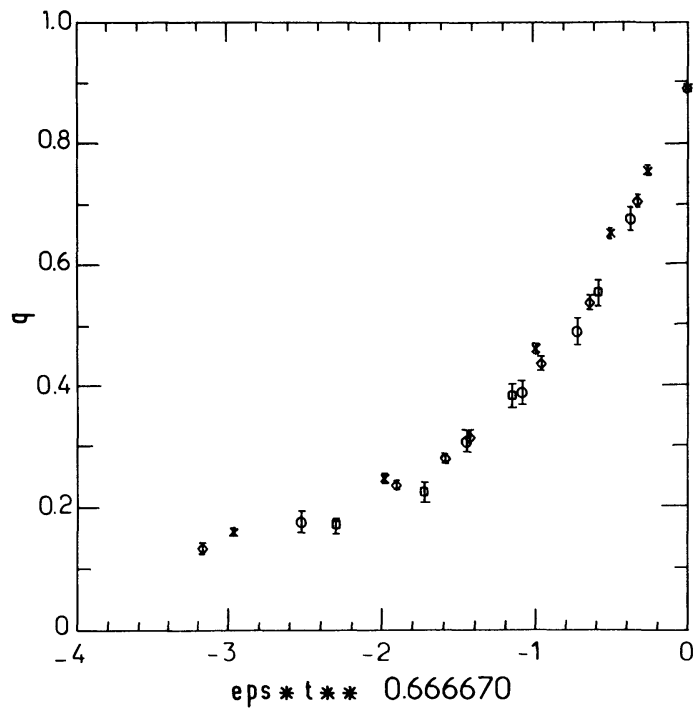

b)

Fig. 1 - a) Average overlap between two copies of the polymer in the same realization of the potential, coupled through a local potential of strength $\varepsilon$, versus $\varepsilon$ ( $\varepsilon$ negative corresponds to a repulsion). Polymers of different lengths are represented by different symbols. The lengths studied are $t=31$ (cross), 63 (diamond), 127 (octogon), 255 (square). The number of samples studied to perform the average is respectively, for each of the sizes : $1000,200,200,200$. The inverse temperature is $\beta=7$. b) Same data plotted versus the variable $\varepsilon t^{2 / 3}$.

energy of the system. It is like a transverse uniform electric field acting on the charged head (endpoint) of the polymer. In the mean field theory (on the tree) it is easy to see that there exists a critical (de Almeida Thouless like) line $T_{\mathrm{c}}(h)$ such that for $T<T_{\mathrm{c}}(h)$ the system is in its glassy phase. The equation for $T_{\mathrm{c}}(h)$ is identical to that found in the REM - simplest spin glass [7, 8]. In one dimension one does not expect such an effect, but we will see that interesting behaviour takes place in the limit of small values of the field. As we have seen the typical lateral extension scales like $\langle x\rangle \sim t^{\nu}$, with $\nu \sim 2 / 3$; furthermore the previous results on coupled systems suggest that the free energy of relevant fluctuations scales like $t^{\tilde{\omega}}$, with say $\tilde{\omega} \sim 1 / 3$. As a consequence it is natural to scale this field like $h=\hat{h} t^{\rho}$ in such a way that $\hat{h} t^{\rho} t^{\nu} \sim t^{\tilde{\omega}}$, i.e. $\rho=\tilde{\omega}-\nu \sim-1 / 3$. Plotting $\langle x\rangle / t^{2 / 3}$ as a function of $h t^{1 / 3}$, we find a linear behaviour which is reasonable since it corresponds to a linear response $\langle x\rangle \sim a h t$ for small $h$. This linear behaviour in $h$ and $t$ is proven in appendix 1 [25]. The surprise comes from the study of each individual sample. In figure 2 we plot $\langle x\rangle / t^{2 / 3}$ and $\left(\left\langle x^{2}\right\rangle-\langle x\rangle^{2}\right) / t$ versus $h t^{1 / 3}$ for one sample, with $\beta=7$ and $t=1023$. We see that the behaviour is much richer. Inside some intervals of $\hat{h}$ the extension $\langle x\rangle / t^{2 / 3}$ is nearly constant, and it jumps suddenly to some other plateau at some critical values of $\hat{h}$, which fluctuate from sample to sample. The susceptibility $\left(\left\langle x^{2}\right\rangle-\langle x\rangle^{2}\right) / t$ is basically zero inside the plateaux, and possesses high peaks at the critical values of $h$; when averaging over samples these high peaks dominate the average leading to $\overline{\left(\left\langle x^{2}\right\rangle-\langle x\rangle^{2}\right)} \sim t$. The width of the peaks scale as $t^{-a}$ and the height like $t^{1+a}$ in order to ensure this linear behaviour in $t$ for the average susceptibility. As we shall see it is reasonable to assume, and consistent with our simulations that $a \sim \tilde{\omega} \sim 1 / 3$. 


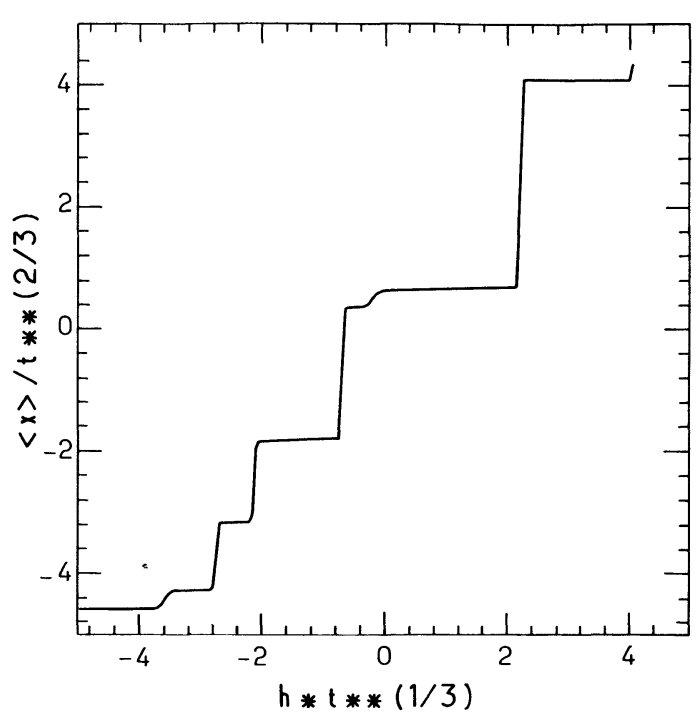

a)

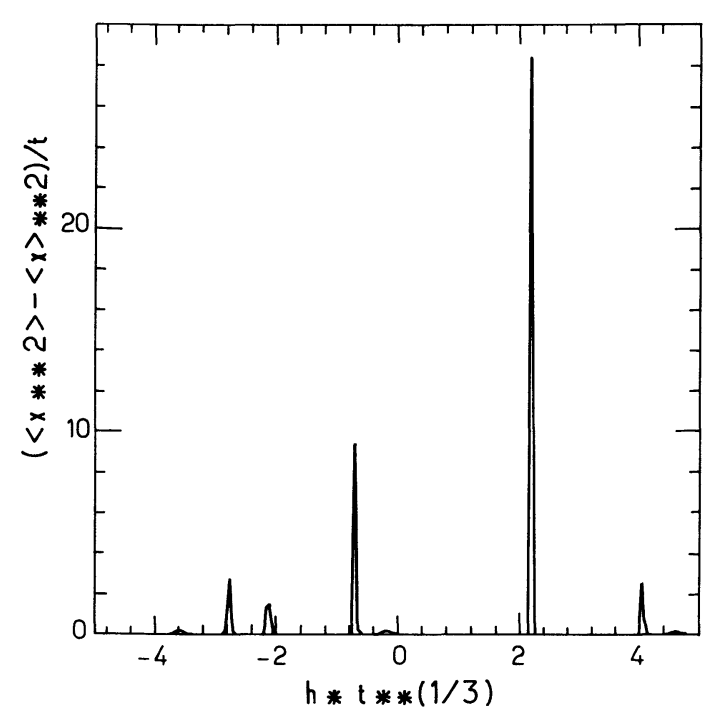

b)

Fig. 2-a) Rescaled average position of the endpoint of the polymer, $\langle x\rangle / t^{2 / 3}$, versus a rescaled transverse electric field, $\hat{h}=h t^{1 / 3}$. The data is from one single sample of length $t=1023$, at inverse temperature $\beta=7$. b) Rescaled susceptibility $\left(\left\langle x^{2}\right\rangle-\langle x\rangle^{2}\right) / t$, versus the rescaled field, for the same sample.

This analysis of the response to a field is interesting since it gives information to what happens in zero field. Concerning the susceptibility $\chi=\left\langle x^{2}\right\rangle-\langle x\rangle^{2}$, we expect that for almost every sample (i.e. with a probability going to one when $t \rightarrow \infty$ ), $\chi / t$ will be zero, but occasionally (with a probability $t^{-\tilde{\omega}}$ ), there will exist a sample where $h=0$ is a critical value of $h$, and therefore $\chi \sim t^{1+\tilde{\omega}}$, so that the average susceptibility $\chi$ is linear in $t$ when $t \rightarrow \infty$. In order to check this, and to compute the typical susceptibility (which must be quite different from the average one), we have computed numerically the values of $\left\langle x^{2}\right\rangle-\langle x\rangle^{2}$ for several thousands of samples with sizes ranging from $t=31$ to 1023 . Letting aside the tail of the distribution corresponding to rare samples (on which the statistics in naturally poor), we have found that the typical susceptibility scales like $t^{2 \mu}$, where $\mu$ is in the range $0-0.2$. In figure 3 we plot the probability that $\chi / t^{0.22}$ be less than $c$, for various sizes $t$. For $c$ not too large this probability is independent of $t$. Of course when $c$ becomes large the finite size effects become important. From figure 3 we conjecture that the limit when $t \rightarrow \infty$ of the probability that $\left(\left\langle x^{2}\right\rangle-\langle x\rangle^{2}\right) / t^{2 \mu}=y$ is a function $P(y)$, with $\int_{0}^{\infty} P(y) \mathrm{d} y=1$. The fact that $\bar{\chi}$ is dominated by rare events corresponds to a tail of $P$ at large $y$, which will behave like $P(y) \underset{y \rightarrow \infty}{\sim} y^{-\rho}, \rho=(5-3 \mu) /(4-3 \mu)$. A more precise determination of $\mu$ is difficult because of large fluctuations in $\log (\chi)$. Actually when plotting the distribution of $\log (\chi)$, we find a peak of the distribution which is independent of $t$, which would indicate that $\mu \sim 0$. But the tail of the distribution at large values of $\log (\chi)$ increases slightly with $t$, resulting in the effective growth of $\exp \overline{(\log (\chi)})$ like $t^{0.22}$. Much more statistics and larger sizes will be needed to give an accurate value of $\mu$, but in any case it is much smaller than 0.5 , which is the important fact in the above discussion. 


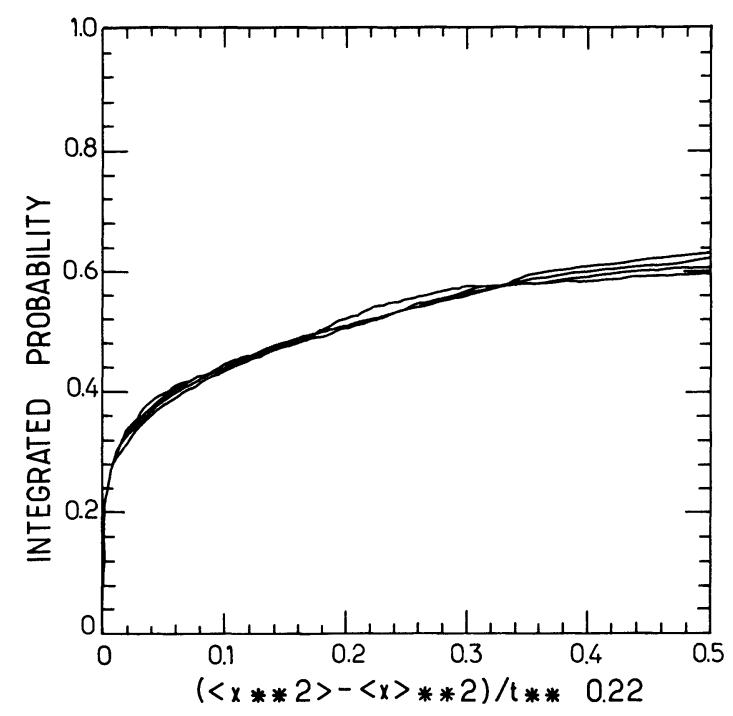

Fig. 3 - Probability that the rescaled susceptibility $\left(\left\langle x^{2}\right\rangle-\langle x\rangle^{2}\right) / t^{0.22}$, be less than a given constant $c$, versus $c$. The data was taken at inverse temperature $\beta=7$, in zero field. The sizes are $t=127,255,511,1023$. The number of samples studied to compute the probability is respectively, for each size : $8000,4000,2000,1000$.

\section{Comparison with the analysis through the replica method.}

As is well known, in order to compute the free energy $F(t)$ and the averaged probability density of the endpoint $P(x, t)$, defined in (4), (5), one can use the replica method and the quantum mechanical representation $[6,19]$. Starting from the partition function $Z(x, t)$ defined in (1) let us introduce :

$$
Z\left(x_{1}, \ldots, x_{n}, t\right)=\overline{Z\left(x_{1}, t\right) \ldots Z\left(x_{n}, t\right)}
$$

then we have : $\quad F=\lim _{n \rightarrow 0}-\frac{T}{n} \log \left(\int \mathrm{d} x_{1} \ldots \mathrm{d} x_{n} Z\left(x_{1}, \ldots, x_{n}, t\right)\right)$

and :

$$
\overline{P(x, t)}=\lim _{n \rightarrow 0} \int \mathrm{d} x_{2} \ldots \mathrm{d} x_{n} Z\left(x, x_{2}, \ldots, x_{n}, t\right) \text {. }
$$

On the other hand, performing explicitly the integration on the random potential in (15), we find for $\hat{Z}\left(x_{1}, \ldots, x_{n}, t\right)=Z\left(x_{1}, \ldots, x_{n}, t\right) \exp \left(-\beta^{2} n t \delta(0) / 2\right)$ a path integral representation which shows that $\hat{Z}$ satisfies the following Schrödinger equation ${ }^{(1)}$ :

$$
-\frac{\partial \hat{Z}\left(x_{1}, \ldots, x_{n}, t\right)}{\partial t}=\left(-\frac{1}{2} \sum_{a} \frac{\partial^{2}}{\partial x_{a}^{2}}-\beta^{2} \sum_{a<b} \delta\left(x_{a}-x_{b}\right)\right) \hat{Z}\left(x_{1}, \ldots, x_{n}, t\right)
$$

(1) Here we use the continuous formulation which is more compact. A precise meaning can be given to all quantities by going to a discretized version of the model, similar to the one used in (11)-(12). Then the infinite constant $\delta(0)$ which appears in the definition of $\hat{Z}$ gets regularized. This constant leads to a term behaving like $-c^{t} / T$ in the free energy density. 
with the limit condition $\hat{Z}\left(x_{1}, \ldots, x_{n}, t=0\right)=\prod_{a=1}^{n} \delta\left(x_{a}\right)$. Therefore $\hat{Z}$ is the Green's function of a system of $n$ particles interacting through an attractive $\delta$ function potential. This suggests to write

$$
\hat{Z}\left(x_{1}, \ldots, x_{n}, t\right)=\sum_{\alpha} \psi_{\alpha}\left(x_{1}, \ldots, x_{n}\right) \psi_{\alpha}^{*}(0, \ldots, 0) \mathrm{e}^{-E_{\alpha} t}
$$

where $\psi_{\alpha}\left(x_{1}, \ldots, x_{n}\right)$ are eigenstates of the $n$ body Hamiltonian (18), and $E_{\alpha}$ are the corresponding energies. For $t$ large (19) should be dominated by the ground state $\left({ }^{2}\right)$, which is at least for $n$ integer and nonzero the Bethe Ansatz wave function :

$$
\psi_{0}\left(x_{1}, \ldots, x_{n}\right)=c^{t} \mathrm{e}^{-\beta^{2} / 2 \sum_{a<b}\left|x_{a}-x_{b}\right|} .
$$

This fact has been used by Kardar to show that the fluctuations of free energies from sample to sample scale as :

$$
\bar{F}^{2}-\bar{F}^{2} \sim t^{2 \omega}, \quad \omega \sim 1 / 3
$$

which in turn implies through an indirect argument that $\nu=2 / 3$.

In order to obtain $P(x, t)$ directly, we need to compute the asymptotic Green's function at large times and therefore to sum over low lying states in (19). A natural suggestion would be to sum over the continuous excitation spectrum corresponding to the centre of mass motion :

$$
\hat{Z}\left(x_{1}, \ldots, x_{n}, t\right)=c_{n} \int \mathrm{d} p \exp \left(-\frac{p^{2} t}{2 n}+\frac{i p}{n} \sum_{a=1}^{n} x_{a}-\frac{\beta^{2}}{2} \sum_{a<b}\left|x_{a}-x_{b}\right|\right) .
$$

The corresponding distribution of endpoint positions, $\bar{P}(x, t)$, is computed in the appendix 2 . For any non zero $n$ and any positive time, we can compute (see the appendix 2 ) the typical drift :

$$
\overline{\left\langle x^{2}\right\rangle}=\frac{\int \mathrm{d} x_{1} \ldots \mathrm{d} x_{n} x_{1}^{2} \hat{Z}\left(x_{1}, \ldots, x_{n}, t\right)}{\int \mathrm{d} x_{1} \ldots \mathrm{d} x_{n} \hat{Z}\left(x_{1}, \ldots, x_{n}, t\right)}
$$

unfortunately the result is :

$$
\overline{\left\langle x^{2}\right\rangle}=\frac{t}{n}+\frac{2}{\lambda^{2} n^{2}}\left(\frac{\pi^{2}}{6}-\psi^{\prime}(n)\right)
$$

and cannot be continued to $n=0$ in a straightforward way (an attempt of such a continuation can be found in [23]). Actually the result (24), which is derived exactly from the assumed Green's function (22), imposes a relation between the time $t$ and the number $n$ of replicas : for $n$ small, one needs $t n^{3}>1$. The existence of a crossover regime where $t \rightarrow \infty, n \rightarrow 0$, and $t n^{3} \sim 1$, suggests that there should be some relevant excited states of the Hamiltonian (18), with an excitation energy of order $n^{3}$, which will contribute to (19) in the limit of $n \rightarrow 0$. The existence of such states hs been pointed out recently by Parisi [15]. He considered

(2) This is not so clear in the $n \rightarrow 0$ limit, there exists a solvable counter example where the limits $t \rightarrow \infty$ and $n \rightarrow 0$ do not commute [13]. 
a state built of two groups of $n / 2$ particles, where the wave function inside each group is of the Bethe type (20), while the center of masses of each group are widely separated. For $n$ small the excitation energy of such a state is indeed of order $n^{3}$. This state is also the relevant one when one uses two copies of the polymer with repulsive interactions. The $n / 2$ replicas of the first copy build one group and the $n / 2$ replicas of the second copy build the second group. As the center of masses of the groups need to be widely separated in order to have an eigenstate of the $n$-body Hamiltonian, this explains the fact (14) that the overlap between the copies vanishes in the case of repulsive interaction.

Clearly, to get the correct asymptotic form of the Green's function, we need to include in (19) such excited states. So far we have not succeeded in getting an explicit expression for $\bar{P}(x, t)$ from this resummation. On the other hand, Parisi has recently proposed a simple Ansatz for the Green's function [15], which takes the following form :

$$
Z\left(x_{1}, \ldots, x_{n}, t\right)=c^{t} \mathrm{e}^{-\frac{1}{2 t} \sum_{a=1}^{n} x_{a}^{2}-\frac{\beta^{2}}{2} \sum_{a<b}\left|x_{a}-x_{b}\right|} .
$$

This predicts :

$$
\overline{P(x, t)}=\int \mathrm{d}[\phi] \mathrm{e}^{-\frac{1}{2 \beta^{2}} \int(\mathrm{d} \phi / \mathrm{d} y)^{2} \mathrm{~d} y} P_{\phi}(x)
$$

with :

$$
P_{\phi}(x)=\frac{\mathrm{e}^{-x^{2} / 2 t+\phi(x)}}{\int \mathrm{d} y \mathrm{e}^{-y^{2} / 2 t+\phi(y)}}
$$

Furthermore, the joint probability for arriving at positions $x_{1}, \ldots, x_{k}$ is found in this Ansatz equal to:

$$
\frac{\overline{Z\left(x_{1}, t\right)}}{Z(t)} \cdots \frac{\overline{Z\left(x_{k}, t\right)}}{Z(t)}=\int \mathrm{d}[\phi] \mathrm{e}^{-\frac{1}{2 \beta^{2}} \int(\mathrm{d} \phi / \mathrm{d} y)^{2} \mathrm{~d} y} P_{\phi}\left(x_{1}\right) \ldots P_{\phi}\left(x_{k}\right)
$$

Therefore to each sample there should correspond a realization of the random potential $\phi(x)$ drawn with the measure $\mathrm{e}^{-\frac{1}{2 \beta^{2}} \int(\mathrm{d} \phi / \mathrm{d} y)^{2} \mathrm{~d} y}$, and the corresponding probability density of $x$ for this given sample should be given by formula (27).

Actually one can change variables in (27) : defining $\hat{x}$ by $x=\hat{x} t^{2 / 3}$ and $\hat{\phi}(\hat{x})=\phi(x) t^{1 / 3}$, one gets :

$$
P_{\hat{\phi}}(\hat{x})=\frac{\mathrm{e}^{-t^{1 / 3}\left(\hat{x}^{2} / 2+\hat{\phi}(\hat{x})\right)}}{\int \mathrm{d} \hat{y} \mathrm{e}^{-t^{1 / 3}\left(\hat{y}^{2} / 2+\hat{\phi}(\hat{y})\right)}}
$$

and $\hat{\phi}$ has the same distribution, $\mathrm{e}^{-\frac{1}{2 \beta^{2}} \int(\mathrm{d} \hat{\phi} / \mathrm{d} y)^{2} \mathrm{~d} y}$, as the original $\phi$. The distribution (29) implies that, at large times, $\hat{x}$ gets trapped into the minimum of the function $\hat{x}^{2} / 2+\hat{\phi}(\hat{x})$, where $\hat{\phi}(\hat{x})$ is nothing but a random walk in $\hat{x}$ space. It is amusing to notice $\left.{ }^{3}\right)$ that this model

(3) We thank J. P. Bouchaud for pointing out reference [20] to us. 
has been introduced as a toy model for the study of random field problems, and the relations like $\overline{\left\langle x^{2}\right\rangle} \sim t^{4 / 3}$ and $\overline{\left\langle x^{2}\right\rangle-\langle x\rangle^{2}} \sim t$ can be deduced exactly from the rigorous analysis of this toy model performed in [20,21].

We have tried to test Parisi's Ansatz further by comparing (29) with numerical results. First of all we argued in the previous section that the typical value of $\left\langle x^{2}\right\rangle-\langle x\rangle^{2}$ is $t^{2 \mu}, \mu \sim 0.0-0.2$ rather than $t$. Simulations of (29) favour a scaling of $\left\langle\hat{x}^{2}\right\rangle-\langle\hat{x}\rangle^{2} \sim$ $\frac{1}{t^{4 / 3-2 \tilde{\mu}}}$, with $\tilde{\mu}$ in the range 0-0.16. (Again there are large fluctuations in the distribution of $\log (\chi)$, similar to the case of the polymer problem.) This is compatible with the equality $\tilde{\mu}=\mu$, but the uncertainties on the values of these two exponents are too large for this to be fully conclusive. We have also computed $Z(x, t)$ for several hundreds of samples of sizes between 63 and 2047 . Figure $4 \mathrm{a}$ contains a plot of $\overline{\log (Z(x, t))-\log (Z(0, t))]} / t^{1 / 3}$ versus $x / t^{2 / 3}=\hat{x}$. According to both Ansätze (22) and (29) this should behave like $c^{t} \hat{x}^{2}$. (The $c^{t}$ comes from the discretization procedure.) This is precisely what is found : for large times the data points sit on a parabola for $|\hat{x}|<5$. (Of course for larger values of $\hat{x}$ the finite size effects are much more important.) Figure $4 \mathrm{~b}$ contains a plot of $\left([\overline{\log Z(x, t)-\log Z(0, t)}]^{2}-[\overline{\log Z(x, t)-\log Z(0, t)}]^{2}\right) /\left(\beta t^{1 / 3}\right)^{2}$ versus $\hat{x}$. From Parisi's Ansatz (29) this should behave like $c^{t}|\hat{x}|$. This behaviour is obtained for small values of $|\hat{x}|(|\hat{x}| \leqslant 2)$, but it saturates for larger values of $|\hat{x}|$. This saturation becomes more apparent when increasing the size of the system (the data in Fig. $4 \mathrm{~b}$ correspond to sizes 511 and 1023 for which the finite size effects in the range $|\hat{x}| \leqslant 5$ become quite small with respect to the fluctuations).

Therefore the Ansatz (29) seems to be an approximation which may be valid only at relatively small values of $|\hat{x}|$.

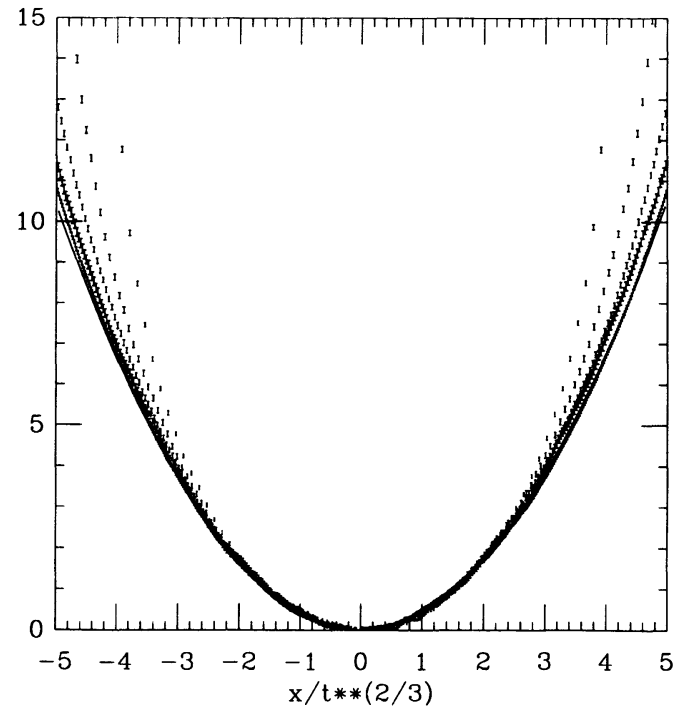

a)

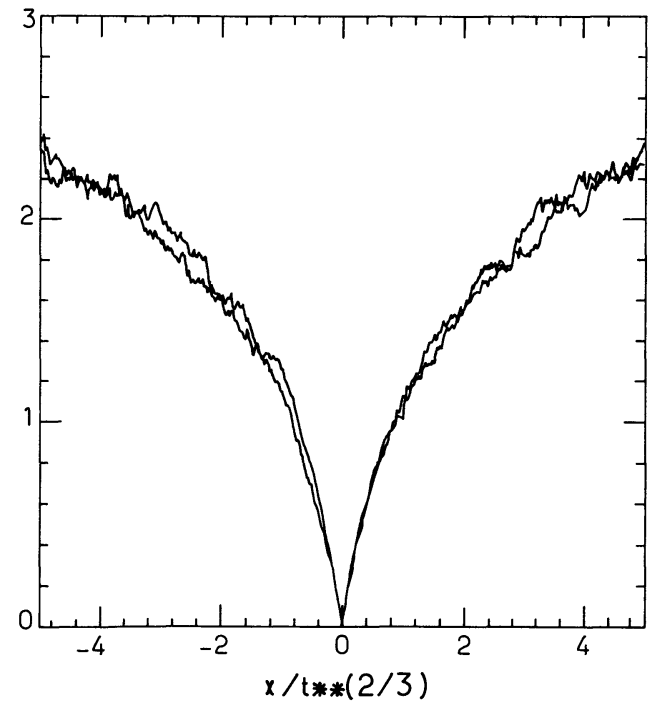

b)

Fig. 4 - a) $-\overline{(\log Z(x, t)-\log Z(0, t))} /\left(\beta t^{1 / 3}\right)$ versus $\hat{x}=x / t^{2 / 3}$. Data taken at inverse temperature $\beta=7$, in zero field. From top to bottom, the curves correspond to polymers of lengths $t=63,127,255,511,1023$. The number of samples studied range between 200 to 1000 . The full curve is the best quadratic fit to the experimental data of $t=1023$, given by: $0.0039+0.014 \hat{x}+$ $0.418 \hat{x}^{2}$. b) Fluctuations, $\left([\overline{\log Z(x, t)-\log Z(0, t)}]^{2}-[\overline{\log Z(x, t)-\log Z(0, t)}]^{2}\right) /\left(\beta t^{1 / 3}\right)^{2}$ versus $\hat{x} .1000$ samples of sizes 511 and 1023 , at $\beta=7$, in zero field. 


\section{Some conjectures on the physical interpretation of the numerical results.}

One of the main results of the previous sections is the instability of the polymer system with respect to an arbitrarily small repulsion between copies. This indicates the existence of several pure states in this system. Here we shall not attempt to give a precise mathematical definition of these pure states, but we shall use this notion in a phenomenological way $\left({ }^{4}\right)$. A state should correspond to a bunch of locally favoured paths, separated from the other states by free energy barriers. (The existence of families of local optimal paths has also been conjectured by Zhang [24], on the basis of an analysis of the fist excited path.) We thus conjecture that there exist such states; the state $\alpha$ has a free energy $F_{\alpha}$ which scales as :

$$
F_{\alpha}=F t+f_{\alpha} t^{\tilde{\omega}}
$$

where $F$ is the free energy density which is the same for all states and the state dependent corrections scale as $t^{\tilde{\omega}}$. (Such a picture is known to exist in mean field, with $\tilde{\omega}=0$.) The Gibbs measure should then be decomposed into the contributions of the states; for any observable $O$ :

$$
\langle O\rangle=\sum_{\alpha} P_{\alpha}\langle O\rangle_{\alpha}
$$

Let us first show how this can explain the results obtained when coupling two copies. In the limit of a small coupling, the average overlap between the copies can be written as :

$$
q(\varepsilon) \sim \frac{\sum_{\alpha, \gamma} \exp \left[-\beta t^{\omega}\left(f_{\alpha}+f_{\gamma}\right)+\beta \varepsilon t q_{\alpha \gamma}\right] q_{\alpha \gamma}}{\sum_{\alpha, \gamma} \exp \left[-\beta t^{\omega}\left(f_{\alpha}+f_{\gamma}\right)+\beta \varepsilon t q_{\alpha \gamma}\right]}
$$

where :

$$
q_{\alpha \gamma}=\left\langle\int_{0}^{t} \mathrm{~d} \tau \delta(x(\tau)-y(\tau))\right\rangle_{x \in \alpha, y \in \gamma} .
$$

The crossover regime is found when $\varepsilon t \sim t^{\tilde{\omega}}$, i.e. $\varepsilon \sim \hat{\varepsilon} t^{\tilde{\omega}-1}$. Such a crossover was precisely found in the simulations of section 2 , and these gave an exponent $\tilde{\omega}$ in the range $0.2-0.4$. The assumption that $\tilde{\omega}=1 / 3$ which we have used in this paper is the simplest one since the sample to sample fluctuations of the free energy are known to scale with the exponent $\omega=1 / 3$ and we are just making the reasonable assumption that $\omega=\tilde{\omega}$ (another argument indicating that $\tilde{\omega}=2 \nu-1$ will be given in the next paragraph). However a more precise direct determination of $\tilde{\omega}$ would be welcome. It is worth exploring further how the above equation (32) can account for the data of figure 1. This equation holds for one given sample. For $\hat{\varepsilon} \rightarrow 0$ the sum over states is dominated by the ground state, say $\alpha=0$, with $f_{0}=0$ by a proper choice of normalizations. In this limit the overlap $q(\hat{\varepsilon})$ tends towards the

$\left({ }^{4}\right)$ Let us point out one necessary condition on the definition of pure states : In order to be able to write equations like (31) and (32) we need some kind of clustering property of the states, in the sense that the joint probability distribution for a polymer to be at point $x$ at time $t$ and at point $x^{\prime}$ at time $t^{\prime}$ must factorize inside each state :

$$
\left\langle\delta(x(t)-x) \delta\left(x\left(t^{\prime}\right)-x^{\prime}\right)\right\rangle_{\alpha} \underset{\left|t-t^{\prime}\right| \rightarrow \infty}{\sim}\langle\delta(x(t)-x)\rangle_{\alpha}\left\langle\delta\left(x\left(t^{\prime}\right)-x^{\prime}\right)\right\rangle_{\alpha} .
$$


self-overlap of the state, $q_{00}$. When $\hat{\varepsilon}$ is negative (repulsive interaction) and large enough, the overlap will jump to $q_{0 \gamma}$, where $\gamma$ is the state such that $|\hat{\varepsilon}|\left(q_{00}-q_{0 \gamma}\right)-f_{\gamma}$ is maximal (assuming that the self overlap of the states are all equal, otherwise the pair of states which dominates (32) might not contain the lowest state). Therefore when $\hat{\varepsilon}$ decreases we expect for $q(\hat{\varepsilon})$ a discontinuous curve, with jumps taking place whenever the gain in free energy from the two copies of the polymer taking paths which have smaller overlap compensates for the loss from at least one polymer taking an excited path. Such a discontinuous curve is found when simulating one sample (although the plateaux are not really constant, which might signal more subtle effects). But the curve in figure 1 was obtained by averaging over many samples, and as the values of the $f_{\gamma}$ at least fluctuate from one sample to another, the average gives back the continuous curve of figure $1 \mathrm{~b}$. The nonzero slope of the curve at $\varepsilon \rightarrow 0^{-}$tells that there is a finite probability of having an arbitrarily small gap between the first excited pure state and the fundamental one. It is difficult to extract more information from this curve without some further assumption on the overlaps of the states. We shall not expand here on the various possibilities. Let us just mention a simple possibility, which is the one which takes place in the mean field theory, and in expansions around the mean field for large dimensions [12]: if the overlaps can just take two values, with $q_{\alpha \alpha}=q$ and $q_{\alpha \gamma}=0$, $\alpha \neq \gamma$, then the curve $q(\hat{\varepsilon})$ of figure $1 \mathrm{~b}$ is simply related to the distribution $\Pi(\Delta f)$ of the gap free energy (rescaled difference between the free energy of the first excited state and the free energy of the lowest lying pure state $\left.\Delta f=\left(F_{1}-F_{0}\right) / t^{\tilde{\omega}}\right)$ :

$$
\overline{q(\hat{\varepsilon})}=q \int_{\hat{\varepsilon} q}^{\infty} \Pi(\Delta f) d \Delta f .
$$

As for the results on the susceptibility (Figs. 2, 3), they can also be accounted for if one supposes that the susceptibility inside each state scales as $t^{2 \mu}, \mu<1 / 2$ :

$$
\chi_{\alpha}=\left\langle x^{2}\right\rangle_{\alpha}-\left(\langle x\rangle_{\alpha}\right)^{2} \sim t^{2 \mu} .
$$

For most samples, the Gibbs average is dominated by the lowest pure state $\alpha_{0}$ and therefore the full Gibbs susceptibility is equal to $\chi_{\alpha_{0}}$. However occasionally it may happen that a sample will have two nearly degenerate pure ground states, with $\Delta f \sim t^{-\tilde{\omega}}$. This will be the case for a fraction of the samples of order $t^{-\tilde{\omega}}$; for such a sample the Gibbs susceptibility must be of order $t^{2 \nu} \sim t^{4 / 3}$ (assuming that the typical transverse fluctuation for each pure state scales as $t^{\nu}$ ). These rare samples will therefore dominate the average susceptibility, leading to a Gibbs susceptibility $\bar{\chi} \sim t^{2 \nu-\tilde{\omega}}$, which gives the correct scaling demonstrated in appendix 1 $(\bar{\chi} \sim t)$ if $2 \nu-\tilde{\omega}=1$.

\section{Summary and perspectives.}

To summarize in a few words, we have found numerically two main results :

- an infinitesimal (but extensive in the thermodynamic sense) local repulsion between two identical copies of the polymer system yields an instability;

- coupling a transverse electric field to the endpoint of the polymer, the corresponding susceptibility scales as $t^{2 \mu}$ for generic samples, with $\mu \sim 0.0-0.2$. The sample averaged susceptibility is dominated by rare events and it has been shown to scale as $t$.

One possible interpretation of these results would be the existence of several pure states, such that :

- the typical differences between the free energies of the states differ by terms which grow with the length of the polymer as $t^{\tilde{\omega}}$, with $\tilde{\omega}=1 / 3$; 
- within a given state $\alpha$ the transverse extension scales as $\langle x\rangle_{\alpha} \sim t^{2 / 3}$, and the susceptibility scales as $\left\langle x^{2}\right\rangle_{\alpha}-\left(\langle x\rangle_{\alpha}\right)^{2} \sim t^{2 \mu}$.

If this interpretation is correct, the situation is as follows : for almost all samples, there is one state which dominates the Gibbs average. For exceptional samples (a fraction $\sim t^{-1 / 3}$ of all the samples), there are two quasi degenerate states, the free energies of which differ only by terms of order 1 . These samples give the dominant contribution to the average susceptibility. Nevertheless, for all samples there are several states and the resulting phase is very similar to a spin glass phase. The major difference is the fact that $\tilde{\omega} \geqslant 0$, while in the mean field theory of spin glasses and of random directed polymers $\tilde{\omega}=0$. But these states exist, they have the same free energy density and each of them can be obtained and stabilized through a quench from high temperatures. From this point of view, it seems that the major effect of having $\tilde{\omega}>0$ is the technical fact that the Gibbs average is generically dominated by one single state.

The remaining open problems are numerous and fascinating. From the analytical point of view we have seen that replica symmetry breaking (i.e. the breaking of Bose symmetry) is necessary to describe the above effects. However a full study of the Green's function of a system of zero particles interacting through delta function potential in one dimension is necessary to solve the problem. Another challenge is of course to try to prove or disprove the above speculations on the physical nature of this phase. The notion of several coexisting pure states seems to be quite useful at least from a phenomenological point of view. A precise mathematical definition of the pure states in this context would be quite important. The extension of the type of analysis carried out here to higher dimensions would also be welcome. One interesting effect which one could try to check numerically in $2+1$ and $3+1$ dimensions is the existence of an instability line of the de Almeida Thouless type when coupling the endpoint of the polymer to a transverse electric field.

\section{Acknowledgments.}

I am indebted to B. Derrida for numerous helpful suggestions and comments, and to G. Parisi for an illuminating discussion. I would also like to thank J. P. Bouchaud, D. S. Fisher and D. J. Gross for useful interactions. The numerical simulations were carried out on the Convex C1 at ENS and on SUN workstations. I thank J. P. Nadal and G. Weisbuch for providing me plenty computer time on their sparcstation.

\section{Appendix 1.}

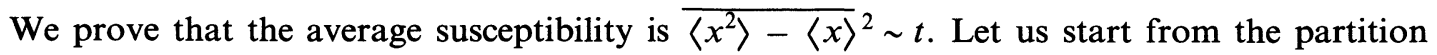
function in the presence of a field $h$, for one sample with potential $V$ :

$$
Z_{V}(h, t)=\int_{x(0)=0} \mathrm{~d}[x] \exp \left(-\frac{1}{2} \int_{0}^{t} \dot{x}^{2}(\tau) \mathrm{d} \tau-\beta h x(t)-\beta \int_{0}^{t} V(x(\tau), \tau) \mathrm{d} \tau\right) .
$$

Using the change of variables $y(\tau)=x(\tau)+\beta h \tau$, this can be rewritten as :

$$
Z_{V}(h, t)=\mathrm{e}^{\frac{\beta^{2} h^{2}}{2} t} \int_{y(0)=0} \mathrm{~d}[y] \exp \left(-\frac{1}{2} \int_{0}^{t} \dot{y}^{2}(\tau) \mathrm{d} \tau-\beta \int_{0}^{t} V(y(\tau)-\beta h \tau, \tau) \mathrm{d} \tau\right) .
$$


From which one deduces :

$$
\log Z_{V}(h, t)=\frac{\beta^{2} h^{2}}{2} t+\log Z_{\tilde{V}}(h=0, t)
$$

where $\tilde{V}(x, \tau)=V(x-\beta h \tau, \tau)$. As $\tilde{V}$ has the same distribution as $V$, we get after sample averaging :

$$
\left.\left.\overline{\log Z_{V}(h, t}\right)=\frac{\beta^{2} h^{2}}{2} t+\overline{\log Z_{V}(h=0, t}\right) .
$$

The second derivative of this expression with respect to $\beta h$ is :

$$
\overline{\left\langle x^{2}\right\rangle-\langle x\rangle^{2}}=t \text {. }
$$

This proves the result for the continuous model. It would be interesting to use (A1.3) to compute the typical susceptibility, but we have not succeeded in doing so.

\section{Appendix 2.}

We compute the distribution $\bar{P}(x, t)$ obtained from the ground state wave function (20), including the degree of freedom of the center of mass motion. The asymptotic behaviour of the Green's function at large times is then approximated by :

$$
\hat{Z}\left(x_{1}, \ldots, x_{n}, t\right)=c_{n} \int \mathrm{d} p \exp \left(-\frac{p^{2}}{2 n} t+i p \frac{1}{n} \sum_{a} x_{a}-\lambda \sum_{a<b}\left|x_{a}-x_{b}\right|\right)
$$

(with $\lambda=\beta^{2} / 2$ ), and we compute :

$$
P_{n}(x, t)=\int \mathrm{d} x_{2}, \ldots, \mathrm{d} x_{n} \hat{Z}\left(x, x_{2}, \ldots, x_{n}, t\right) .
$$

The average distribution of the end point position, $\bar{P}(x, t)$, should be obtained eventually from the $n \rightarrow 0$ limit of the previous formula. However because of the problems involved with this limit we first compute $P_{n}$. The normalization constant $c_{n}$ will be obtained from the condition :

$$
\int \mathrm{d} x P_{n}(x, t)=1
$$

Choosing an ordering of the particles' positions we get :

$$
\begin{aligned}
P_{n}(x, t)=c_{n} n ! \int \mathrm{d} p \mathrm{e}^{-n t p^{2} / 2} \sum_{k=1}^{n} \int_{x_{1} \leqslant \ldots \leqslant x_{n}} \mathrm{~d} x_{1}, \ldots, \mathrm{d} x_{n} \delta\left(x_{k}-x\right) \times & \times \exp \left(\sum_{\ell=1}^{n}\left(p_{\ell}+i p\right) x_{\ell}\right)
\end{aligned}
$$

where :

$$
p_{\ell}=(n+1-2 \ell) \lambda .
$$

Performing the integrations over the variables $x_{\ell}(\ell \neq k)$ gives :

$$
P_{n}(x, t)=c_{n} n \int \mathrm{d} p \mathrm{e}^{-n t p^{2} / 2+i p n x} \lambda^{1-n} S_{n}
$$


where :

$$
S_{n}=\frac{\Gamma(1-n-i p / \lambda)}{\Gamma(n-i p / \lambda)} \sum_{k=1}^{n}(-1)^{k-1} \frac{\Gamma(n)}{\Gamma(k) \Gamma(n-k+1)} \frac{\Gamma(k-i p / \lambda)}{\Gamma(k-n-i p / \lambda)} .
$$

In order to compute $S_{n}$ we first rewrite (with $k^{\prime}=k-1$ integer) :

$$
\frac{1}{\Gamma\left(k^{\prime}+1-n-i p / \lambda\right)}=(-1)^{k^{\prime}} \frac{\Gamma\left(n-k^{\prime}+i p / \lambda\right)}{\Gamma(n+i p / \lambda) \Gamma(1-n-i p / \lambda)} .
$$

From (A2.7) and (A2.8), introducing integral representations of the $\Gamma$ functions which remain at the numerator, we get :

$$
S_{n}=\frac{1}{\Gamma(n-i p / \lambda) \Gamma(n+i p / \lambda)} \int_{0}^{\infty} \mathrm{d} t \int_{0}^{\infty} \mathrm{d} u \mathrm{e}^{-(t+u)}(t+u)^{n-1}\left(\frac{u}{t}\right)^{i p / \lambda} .
$$

In the integral we change variables from $t, u$ to $x, z$, where $t=x /\left(1+\mathrm{e}^{z}\right)$ and $u=t \mathrm{e}^{z}$. This gives eventually:

$$
\begin{aligned}
S_{n} & =\frac{\Gamma(n+1)}{\Gamma(n-i p / \lambda) \Gamma(n+i p / \lambda)} \int_{-\infty}^{+\infty} \mathrm{d} z \frac{\mathrm{e}^{z(1+i p / \lambda)}}{\left(1+\mathrm{e}^{z}\right)^{2}} \\
& =\frac{\Gamma(n+1)}{\Gamma(n-i p / \lambda) \Gamma(n+i p / \lambda)} \frac{p \pi / \lambda}{\sinh (p \pi / \lambda)} .
\end{aligned}
$$

From (A2.6) and (A2.10) we can now compute the final form of $P_{n}$. The normalisation constant is found equal to : $c_{n}=\Gamma(n) \lambda^{n-1} / 2 \pi n$, and :

$P_{n}(x, t)=\frac{1}{2} \Gamma(n)^{2} n \int \mathrm{d} p \mathrm{e}^{-n t p^{2} / 2+i p n x} \frac{1}{\Gamma(n-i p / \lambda) \Gamma(n+i p / \lambda)} \frac{p / \lambda}{\sinh (p \pi / \lambda)}$.

Clearly the limiting distribution for $n \rightarrow 0$ is ill-defined. Actually we can compute the moments through :$$
\overline{\left\langle x^{2 k}\right\rangle}=\frac{1}{2} \Gamma(n)^{2} \frac{2 \pi}{n^{2 k}}(-1)^{k}\left(\frac{\partial}{\partial p}\right)^{2 k}\left[\frac{\mathrm{e}^{-n t p^{2} / 2}}{\Gamma(n-i p / \lambda) \Gamma(n+i p / \lambda)} \frac{p / \lambda}{\sinh (p \pi / \lambda)}\right]_{p=0} .
$$

This gives for instance :

$$
\begin{aligned}
& \overline{\left\langle x^{2}\right\rangle}=\frac{t}{n}+\frac{2}{\lambda^{2} n^{2}}\left(\frac{\pi^{2}}{6}-\psi^{\prime}(n)\right) \\
& \overline{\left\langle x^{4}\right\rangle}-3 \overline{\left\langle x^{2}\right\rangle^{2}}=\frac{1}{\lambda^{4} n^{4}}\left(2 \pi^{4} / 15-2 \psi^{(3)}(n)\right) .
\end{aligned}
$$

From (A2.13), using the fact that $\psi^{\prime}(n) \sim_{n \rightarrow 0} \pi^{2} / 6-1 / n^{2}$, we find that the distribution makes sense only for $t n^{3}$ larger than one (in the limits $t \rightarrow \infty, n \rightarrow 0$ ). 


\section{References}

[1] Kardar M. and Zhang Y. C., Phys. Rev. Lett. 58 (1987) 2087.

[2] Huse D. A. and Henley C. L., Phys. Rev. Lett. 54 (1985) 2708.

[3] Derrida B. and Spohn H., J. Stat. Phys. 51 (1988) 817.

[4] Kardar M., Parisi G. and Zhang Y. C., Phys. Rev. Lett. 56 (1986) 889.

[5] Huse D. A., Henley C. L. and Fisher D. S., Phys. Rev. Lett. 55 (1985) 2924.

[6] Kardar M., Nucl. Phys. B 290 [FS 20] (1987) 582, and references therein.

[7] Derrida B., Phys. Rev. B 24 (1981) 2613.

[8] Gross D. J. and Mézard M., Nucl. Phys. B 240 (1984) 431.

[9] For a review, see Mézard M., Parisi G. and Virasoro M. A., Spin glass theory and beyond (World Scientific, Singapore) 1987.

[10] Imbrie J. Z. and Spencer T., J. Stat. Phys. 52 (1988) 609 ;

CooK J. and Derrida B., J. Stat. Phys. 57 (1989) 89.

[11] Derrida B. and Golinelli O., Saclay preprint SPhT/89-170, to appear in Phys. Rev. A.

[12] COOK J. and DERridA B., Europhys. Lett. 10 (1989) 195.

[13] PARISI G., Rome preprint, to appear in the Rendiconti dell'Accademia dei Lincei.

[14] Kardar M., Phys. Rev. Lett. 55 (1989) 2923.

[15] PARISI G., Rome University, Preprint ROMF2-89/24.

[16] PARISI G., Phys. Rev. Lett. 50 (1983) 1946.

[17] Huse D. A. and Fisher D. S., J. Phys. A 20 (1987) L997.

[18] Parisi G. and Virasoro M. A., J. Phys. France 50 (1989) 3317.

[19] For a recent review, see M. Kardar's contribution to the 1989 meeting on «New trends in Magnetism ", Recife.

[20] Schulz U., Villain J., Brézin E. and Orland H., J. Stat. Phys. 51 (1988) 1.

[21] Villain J., Semeria B., Lanon F. and Billard L., J. Phys. C 16 (1983) 2588.

[22] Caracciolo S., Parisi G., Patarnello S. and Sourlas N., to appear in Europhys. Lett. (1990).

[23] Bouchaud J. P. and ORland H., in preparation.

[24] Zhang Y. C., Phys. Rev. Lett. 59 (1987) 2125.

[25] This result has also been proven by D. S. Fisher and D. A. Huse (in preparation). 